\title{
Riesgos psicosociales y estrés como predictores del burnout en médicos internos residentes en el Servicio de Urgencias
}

\author{
María Fernández-Prada', Joaquín González-Cabrera², Concepción Iribar-Ibabe 3,4 \\ y José María Peinado 3,4 \\ ${ }^{1}$ Servicio de Medicina Preventiva y Salud Pública, Hospital Universitario Central de Asturias, Oviedo; ${ }^{2}$ Departamento de Psicología de la Educación \\ y Psicobiología, Universidad Internacional de La Rioja, Logroño; ${ }^{3}$ Departamento de Bioquímica y Biología Molecular III e Inmunología; ${ }^{4}$ Instituto de \\ Neurociencias Federico Olóriz. Universidad de Granada, Granada, España
}

\begin{abstract}
Resumen
Objetivo: Evaluar el estrés, los riesgos psicosociales asociados al puesto de trabajo y el burnout en un grupo de residentes en el servicio de urgencias; y analizar qué variables se relacionan y predicen mejor el burnout. Método: Estudio analítico y transversal en el que han participado 42 médicos internos residentes que hacen guardias en el servicio de urgencias del Hospital Universitario San Cecilio, Granada. Se han utilizado la versión adaptada al español de la Perceived Stress Scale para evaluar el estrés, el Maslach Burnout Inventory (MBI) para valorar el desgaste profesional, y el cuestionario adaptado y baremado para la autoevaluación de riesgos psicosociales en el trabajo (CopSoQ-ISTAS21). Resultados: El 78\% de los residentes evaluados se encuentra en la categoría desfavorable o intermedia en todas las dimensiones del CopSoQ-ISTAS21, y destaca que el 90\% presenta una puntuación desfavorable en exigencias psicológicas. Además, en el MBI el $45 \%$ presenta conjuntamente un alto agotamiento emocional y una elevada despersonalización. La dimensión de exigencias psicológicas del ISTAS21 $\beta=0.393$; $p<0.003)$ y las puntuaciones de estrés $(\beta=0.451 ; p<0.001)$ predicen significativamente el agotamiento emocional $\left(r^{2}=0.443\right)$. Por último, el $38 \%$ ha sufrido una amenaza/agresión en el servicio de urgencias. Conclusión: La situación de los residentes parece perjudicial para su desempeño profesional debido a los altos riesgos psicosociales asociados al puesto de trabajo. Las exigencias psicológicas se proponen como un importante predictor del burnout. Se recomiendan intervenciones psicológicas y estructurales con las que mejorar la situación de los residentes que realizan guardias en el servicio de urgencias.
\end{abstract}

PALABRAS CLAVE: Burnout. Estrés. Riesgos psicosociales. Residentes. Guardias.

\begin{abstract}
Objective: To study the stress, the psychosocial risks associated to the job and the burnout, in a group of junior doctors working at the emergency ward; and to analyze what of those variables could predict and are better related with burnout. Methods: Cross-sectional study, with a sample of 42 junior doctors which are on duty in the emergency ward of the University Hospital San Cecilio, Granada (Spain). The Spanish adapted version of the Perceived Stress Scale was used to evaluate stress, the Maslach Burnout Inventory (MBI) to evaluate the professional burnout and the adapted and scaled questionnaire for the self-evaluation of psychosocial risks at work (CopSoQ-ISTAS21). Results: $78 \%$ of the junior doctors are in the unfavorable or intermediate range for all COPSOQ-ISTAS21 dimensions, being particularly relevant that $90 \%$ of them display unfavorable score in psychological demands. In addition, MBI results show that $45 \%$ of our population presents high emotional exhaustion simultaneously to high depersonalization. ISTAS21 psychological demands dimensions $(\beta=0.393 ; p<0.003)$ and stress scores $(\beta=0.451 ; p<0.001)$ significantly predict emotional exhaustion $\left(r^{2}=0.443\right)$. Finally, $38 \%$ of junior doctors ex-
\end{abstract}


perienced a threat/aggression during their work in the emergency ward urgencies. Conclusion: Junior doctors develop its professional activity under adverse circumstances probably due to the high psychosocial risk associated to the job. Psychological demands are suggested as the main predicting factor of burnout. These results indicate the need of psychological and structural interventions in order to improve the professional performance of junior doctors at the emergency ward.

KEY WORDS: Burnout. Psychosocial risk. Stress. Junior doctors. Emergency guards.

\section{Introducción}

En España se incorporan al Sistema Nacional de Salud anualmente un gran número de titulados en medicina, enfermería, farmacia, biología y química, entre otras, que han superado la prueba para acceder a una plaza de formación sanitaria especializada. Concretamente, en la convocatoria de 2014-2015 se presentaron al examen 35,320 titulados para obtener una de las 7,527 plazas ofertadas por el Ministerio de Sanidad, Servicios Sociales e Igualdad ${ }^{1}$. En el ámbito médico, se calcula que en 2014 existían en España 30,051 médicos internos residentes (MIR), de los cuales alrededor de $7,400(24,6 \%)$ correspondían a medicina familiar y comunitaria, y por tanto realizan guardias en el servicio de urgencias².

En términos generales, los MIR constituyen un colectivo con amplios conocimientos teóricos que se incorpora por primera vez a un mercado laboral exigente y competitivo. La carga de trabajo y la responsabilidad que se confiere a esta figura médica puede ser, en algunas ocasiones, desproporcionada con respecto a su experiencia y habilidades. En este sentido, estos médicos representan un grupo potencialmente vulnerable a problemas de estrés, burnout y riesgos psicosociales, sobre todo en entornos donde la necesidad de respuesta inmediata es inherente al puesto $^{3}$. Una parte importante de la investigación se ha centrado preponderantemente en la evaluación del burnout $t^{4} \sin$ evaluar otros constructos como el estrés, la calidad de vida en función de la salud ${ }^{5}$ los riesgos psicosociales asociados al puesto de trabajo, así como la posible predicción de los mismos o la relación entre ellos. Quienes más han profundizado en el tema han relacionado variables sociodemográficas como el número de guardias con mayores niveles de burnout ${ }^{6}$. No obstante, se hace necesario profundizar en los factores personales y profesionales que pueden ayudar a explicar el burnout ${ }^{7}$.

Por otro lado, los riesgos psicosociales y el estrés se están configurando como una de las líneas de actuación prioritarias en salud, de tal forma que la Agencia Europea para la Seguridad y Salud en el
Trabajo se centró en 2014 y 2015 en esos lemas durante las Semanas Europeas para la Seguridad y Salud en el Trabajo ${ }^{8}$. No obstante, hasta la fecha son pocas las publicaciones realizadas sobre personal médico, y muchas menos con médicos en formación. Atendiendo al personal médico de manera global, González-Cabrera, et al. ${ }^{9}$ han puesto de manifiesto las relaciones entre los riesgos psicosociales y el estrés en los médicos de urgencias. Estos autores observan que dicho puesto de trabajo se caracteriza por unas altas exigencias psicológicas (EP), un intermedio control sobre el puesto y un AS desfavorable, y por tanto se trata de un caldo de cultivo ideal para la aparición de estrés laboral. Además, la gravedad de los pacientes que acuden a estos servicios, así como los turnos de guardias de hasta 24 horas, podrían ser factores contribuyentes al desarrollo de este fenómeno. Otros autores también han establecido relaciones entre estas dimensiones psicológicas en otros colectivos, como los funcionarios de prisiones y los docentes ${ }^{10,11}$.

Además, la afectación psicológica y física mostrada por las personas sometidas a elevados niveles de estrés incide negativamente en la salud y en la operatividad comportamental. A su vez, el estrés está estrechamente relacionado con la cantidad de recursos psicosociales que se poseen o se cree poseer para afrontar las exigencias de determinadas situaciones de demanda ${ }^{12}$.

Los objetivos de este trabajo son evaluar la situación psicológica de los MIR en relación con el estrés, los riesgos psicosociales asociados al puesto de trabajo y el burnout, y analizar qué variables se relacionan y predicen mejor el burnout.

\section{Método}

Los participantes fueron 42 MIR del Hospital Universitario San Cecilio (Granada, España), 32 mujeres y 10 hombres, con una media de edad de $26.38 \pm$ 1.71. Cada uno realizó una media de $68.31 \pm 17.71$ horas mensuales en el servicio de urgencias (con un rango de 40-140 horas/mes). 32 fueron de la rama 
médica, 5 quirúrgica y 5 médico-quirúrgica. Hubo 25 residentes de primer año (R1) y 17 de segundo año (R2). En relación con la estructura organizativa, los MIR tuvieron una jornada laboral de mañana (8:0015:00 h), que se podía extender hasta las 8:00 h del día siguiente en concepto de "guardia». El estudio se llevó a cabo entre los meses de febrero y abril de 2012, ambos inclusive. Los criterios de inclusión fueron ser MIR y realizar al menos 40 horas mensuales de guardias en el servicio de urgencias. El único criterio de exclusión aplicado fue haber experimentado un suceso traumático psicológico en los últimos 6 meses. Se llevó a cabo un muestreo no probabilístico de tipo incidental. En el periodo de estudio, 75 MIR (41 R1 y 34 R2) realizaron guardias en el servicio de urgencias. Participó el $52 \%$ de la población diana.

Las variables sociodemográficas, como sexo, edad, tipo de especialidad, año de residencia, número de horas de guardia, amenazas/agresiones físicas 0 verbales recibidas, y otros datos, se muestran en la tabla 1.

Se han empleado tres cuestionarios validados (uno de ellos está baremado para la población española) y autoadministrados:

- Versión adaptada al español de la Perceived Stress Scale (EEP, Escala de Estrés Percibido). La versión original de la EEP consta de 14 ítems que evalúan la percepción del control sobre las demandas del entorno. Se ha utilizado una versión adaptada y validada al español ${ }^{13}$.

- Cuestionario para la autoevaluación de riesgos psicosociales en el trabajo (CopSoQ-ISTAS21, versión corta 1.5) ${ }^{14}$. Está diseñado para identificar, medir y valorar la exposición a seis grandes dimensiones de riesgo de naturaleza psicosocial para la salud en el trabajo: a) EP, como volumen de trabajo en relación al tiempo disponible para realizarlo y la transferencia de sentimientos en el trabajo; b) doble presencia, es decir, la

Tabla 1. Frecuencias relativas o media aritmética ( \pm desviación típica) de las variables sociodemográficas. Se han realizado comparaciones para muestras dependientes $(n=42)$

\begin{tabular}{|c|c|c|c|c|}
\hline \multirow[b]{2}{*}{1} & \multirow[b]{2}{*}{$\begin{array}{l}\text { ¿Ha sufrido alguna agresión y/o amenaza física/verbal } \\
\text { por parte de algún paciente? }\end{array}$} & \multirow{2}{*}{$\begin{array}{l}\text { Frecuencias } \\
\text { Sí }=16 \\
\text { No }=26\end{array}$} & \multicolumn{2}{|c|}{$\begin{array}{c}\text { Media } \\
\text { ( } \pm \text { desviación típica) }\end{array}$} \\
\hline & & & & \\
\hline 2 & ¿Cuántas? & $\begin{array}{l}\text { Una vez }=7 \\
\text { Dos veces }=6 \\
\text { Tres o más }=3\end{array}$ & & \\
\hline 3 & ¿Interpuso contra el agresor alguna actuación legal? & $\mathrm{No}=16$ & & \\
\hline 4 & $\begin{array}{l}\text { ¿Tiene permiso de su servicio para tomarse el saliente } \\
\text { de guardia? }\end{array}$ & $\begin{array}{l}\text { Sí }=39 \\
\text { No }=3\end{array}$ & & \\
\hline 5 & $\begin{array}{l}\text { El día después de la guardia, ¿piensa en los pacientes } \\
\text { que atendió cuestionándose el diagnóstico/tratamiento } \\
\text { prescrito? }\end{array}$ & & $3.54( \pm 1.15)$ & \\
\hline 6 & $\begin{array}{l}\text { ¿Con qué frecuencia suele consultar a compañeros en } \\
\text { cada guardia? }\end{array}$ & & $3.97( \pm 0.80)$ & $\begin{array}{l}t=1.159 \\
p<0.253\end{array}$ \\
\hline 7 & $\begin{array}{l}\text { ¿Con qué frecuencia suele consultar a sus superiores } \\
\text { en cada guardia? }\end{array}$ & & $3.42( \pm 0.96)$ & \\
\hline 8 & $\begin{array}{l}\text { ¿Con qué frecuencia percibe usted que sus dudas } \\
\text { son resueltas adecuadamente al preguntarle a un } \\
\text { compañero? }\end{array}$ & & $3.47( \pm 0.83)$ & $\begin{array}{l}t=0,883 \\
p<0.393\end{array}$ \\
\hline 9 & $\begin{array}{l}\text { ¿Con qué frecuencia percibe usted que sus dudas son } \\
\text { resueltas adecuadamente al preguntarle a un superior? }\end{array}$ & & $3.31( \pm 0,96)$ & \\
\hline 10 & $\begin{array}{l}\text { ¿Con qué frecuencia se siente respaldado por un } \\
\text { compañero ante un problema con un paciente? }\end{array}$ & & $4.04( \pm 0,93)$ & $\begin{array}{l}t=7.051 \\
p<0.001\end{array}$ \\
\hline 11 & $\begin{array}{l}\text { ¿Con qué frecuencia se siente respaldado por su } \\
\text { superior ante un problema con un paciente? }\end{array}$ & & $2.83( \pm 0.88)$ & \\
\hline
\end{tabular}


necesidad de responder simultáneamente a las demandas del empleo y del trabajo doméstico-familiar; c) control sobre el trabajo (CT), o margen de autonomía en la forma de realizar el trabajo y las posibilidades que se dan de aplicar habilidades y conocimientos; d) apoyo social y calidad de liderazgo (AS), como apoyo de los superiores o compañeros en la realización del trabajo; e) estima (EST), reconocimiento personal y respeto que se obtiene en relación con el esfuerzo que se realiza en el trabajo; y f) inseguridad sobre el futuro, entendida como la preocupación por los cambios de condiciones de trabajo no deseadas o su pérdida. Los niveles de exposición pueden clasificarse cualitativamente como desfavorables, intermedios o favorables. Este criterio hace referencia al riesgo para la salud de naturaleza psicosocial que tiene su origen en la organización del trabajo.

- Versión española del Maslach Burnout Inventory $(\mathrm{MBI})^{15}$, que evalúa tres dimensiones: agotamiento emocional (AE), despersonalización (DE) y realización personal (RP). Consta de 22 ítems, cada uno de los cuales se valora con una escala del tipo Likert de 0-6 puntos. Con el fin de obtener comparabilidad en los resultados, la puntuación total del MBI se ha obtenido sumando solo el AE y la DE (excluyendo la escala de RP) ${ }^{7}$.

Se realizaron varias reuniones con los MIR en las que se expusieron las líneas generales de la investigación y se proporcionaron la hoja informativa y el consentimiento informado. Se solicitaba que los cuestionarios fueran rellenados en un solo momento temporal y se daban como únicas instrucciones que leyeran las indicaciones explicitadas en el cuadernillo de respuestas y que respondieran específicamente por su experiencia acumulada en el servicio de urgencias, no en su servicio de origen.

La colaboración fue voluntaria, anónima y desinteresada. El procedimiento ético siguió los estándares propios de la Declaración de Helsinki. Cada participante firmó un consentimiento informado escrito, en el que se incluyó la posibilidad de retirarse en cualquier fase del estudio. El estudio fue aprobado por la Comisión de Ética de la Investigación de la provincia de Granada.

Los análisis estadísticos se llevaron a cabo mediante el programa SPSS $15.0\left(\right.$ IBM $\left.^{\circ}\right)$ y las representaciones gráficas con el Sigmaplot 11.0 (Systat Software ${ }^{\odot}$ ).
Se realizaron los siguientes análisis: 1) comprobación de la distribución normal de la muestra (estadístico de Shapiro-Wilks) y la homogeneidad de las varianzas (prueba de Levene); 2) análisis de frecuencias, medidas de tendencia central y dispersión de la medida; 3) cálculo de las puntuaciones tipificadas para todas las variables donde se establecieron comparaciones o relaciones; 4) $t$ de Student para muestras independientes y dependientes; 5) correlaciones bivariadas de Pearson; 6) análisis de la varianza; 7) estadístico $\chi^{2} ;$ y 8) regresión lineal múltiple por "pasos sucesivos" usando la probabilidad de $\mathrm{F}$ para un valor de entrada de 0.15 y de salida de 0.20. Se consideró significativo un valor de $p<0.05$.

\section{Resultados}

En la tabla 1 se muestran parcialmente las variables sociodemográficas relacionadas con el trabajo en el servicio de urgencias, entre las que destaca que el $38 \%$ de los encuestados manifiesta haber tenido, al menos, una agresión o amenaza. Además, se aprecia la preferencia de los MIR por dirigirse a sus homólogos antes que a sus superiores, especialmente cuando quieren sentirse respaldados en la toma de decisiones $(p<0.001)$.

En relación con las puntuaciones obtenidas en la EEP, nuestros resultados muestran que $14 \mathrm{MIR}$ (el $33,3 \%$ ) se encuentran por encima del percentil 75 , lo que indica que un porcentaje relevante presenta un control reducido sobre las demandas de su entorno.

No hay diferencias significativas entre hombres $y$ mujeres $(t=-1.206 ; p<0.235)$ ni entre $R 1$ y $R 2$ $(t=0.362 ; p<0.719)$. Tampoco las hay entre los distintos tipos de especialidades $\left(F_{2,39}=0.808 ; p<0.453\right)$, entre quienes han sufrido o no una agresión 0 una amenaza $(t=-0.748 ; p<0.450)$ ni entre quienes deciden descansar 0 no el día posterior a la guardia $(t=-0.385 ; p<0.702)$.

En la Fig. 1 se representa el porcentaje de MIR que se encuentran en la categoría desfavorable/bajo, intermedio/medio y favorable/alto para las seis dimensiones del cuestionario ISTAS21 y las tres del MBI. Es importante resaltar que el $78 \%$ se encuentra en la categoría desfavorable o intermedia en todas las dimensiones del ISTAS21. Además, en el MBI el $45 \%$ presenta conjuntamente un alto $A E$ y una alta $D E$. No hay diferencias significativas entre hombres y mujeres en ninguna dimensión del ISTAS21 ni del MBI. Entre 
$\mathrm{R} 1$ y $\mathrm{R} 2$ solo se encontraron diferencias en RP ( $\mathrm{t}=$ -2.434; $p<0.017)$. No hay diferencias en función del tipo de especialidad, descansar o no el día posterior a la guardia o haber sufrido o no una agresión o amenaza.

Los valores medios y las desviaciones típicas de las dimensiones del MBI son: AE, $20.95 \pm 10.52$; DE, $7.33 \pm 5.76 ; y \mathrm{RP}, 37,76 \pm 5,69$.

En la tabla 2 se indica la relación entre la presencia de burnout y diferentes variables sociodemográficas, así como los valores obtenidos tras realizar comparaciones mediante el estadístico $\chi^{2}$. La RP en función del año de residencia ha sido la única comparación significativa $\left(\chi^{2}=6.212 ; p<0.045\right)$.

En cuanto a las variables que se relacionan y predicen el burnout, la tabla 3 muestra las correlaciones parciales entre las puntaciones totales de la EEP, el MBI y el ISTAS21 controlando por edad.

En la tabla 4 aparecen las regresiones lineales múltiples usando como predictores las dimensiones del ISTAS21 y la EEP sobre los criterios de AE, DE y RP. Nuestros resultados muestran que las dimensiones de EP y EST del ISTAS21, junto con la puntuación total de la escala EEP, son los mejores predictores de las dimensiones del $\mathrm{MBI}$ en nuestra muestra.
Por último, si realizamos una regresión lineal utilizando como criterio la puntuación total del MBI y como predictor la cantidad de horas en el servicio de urgencias encontramos que esta no es una variable relevante para predecir el burnout $\left(r^{2}=0.034\right.$; $\beta=-0.185 ; t=-1.160 ; p<0.253)$.

\section{Discusión}

El presente trabajo ha abordado de forma novedosa un constructo de estudio muy discutido en la literatura actual. Se han obtenido indicadores empíricos con los que predecir los niveles de burnout en una muestra de MIR que realizan guardias en el servicio de urgencias. Las altas EP y los altos niveles de estrés son los mejores predictores tanto para el AE como para la RP entre los médicos noveles (tabla 4). Además, la estima predice significativamente la RP. No obstante, si se realiza una evaluación conjunta de las distintas dimensiones del ISTAS21, especialmente las que guardan relación directa con el modelo de demandas-control de Karasek y Theorell ${ }^{16}$, encontramos resultados convergentes con lo expuesto para otros grupos profesionales médicos como los médicos de urgencias: altas EP, un control deficiente sobre la

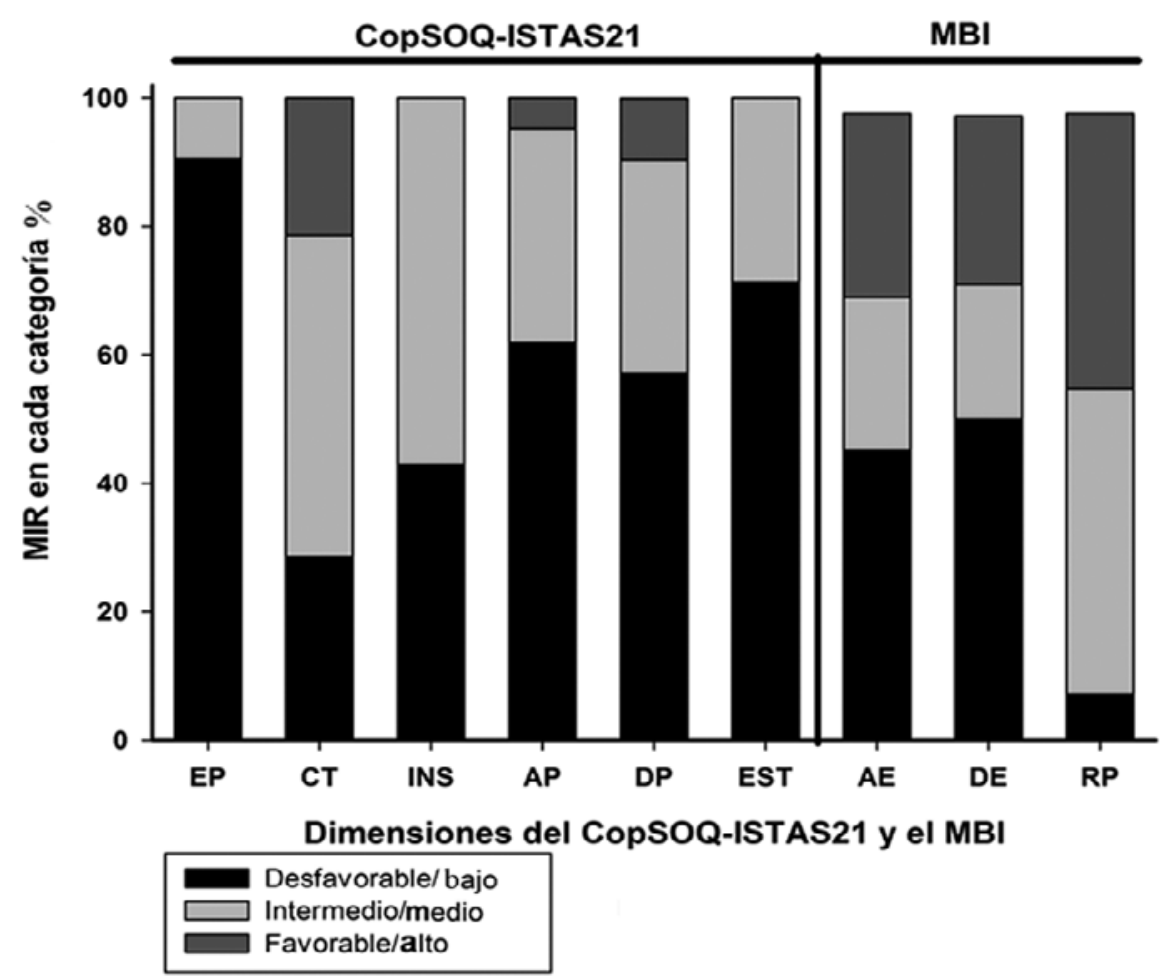

Figura 1. Porcentaje de médicos internos residentes que se encuentran en una posición favorable/intermedia/desfavorable en las dimensiones del CopSOQ-ISTAS21 y el MBI. AE: agotamiento emocional; AP: apoyo social; CT: control del trabajo; DE: despersonalización; DP: doble presencia; EP: exigencias psicológicas; EST: estima personal; INS: inseguridad en el futuro; RP: realización personal. 
Tabla 2. Relación entra la presencia de burnout y diferentes variables sociodemográficas y comparaciones $\chi^{2}(n=42)$

\begin{tabular}{|c|c|c|c|c|c|c|c|c|c|}
\hline & \multicolumn{3}{|c|}{ Agotamiento emocional } & \multicolumn{3}{|c|}{ Despersonalización } & \multicolumn{3}{|c|}{ Realización personal } \\
\hline & \multicolumn{2}{|c|}{ Hombres } & Mujeres & \multicolumn{2}{|c|}{ Hombres } & Mujeres & \multicolumn{2}{|c|}{ Hombres } & Mujeres \\
\hline \multicolumn{10}{|l|}{ Sexo } \\
\hline Bajo & \multicolumn{2}{|c|}{$5(11.9 \%)$} & $15(35.7 \%)$ & \multicolumn{2}{|c|}{$5(11.9 \%)$} & $17(40.5 \%)$ & \multicolumn{2}{|c|}{$1(2.4 \%)$} & $3(7.1 \%)$ \\
\hline Medio & \multicolumn{2}{|c|}{$2(4.8 \%)$} & $8(19 \%)$ & \multicolumn{2}{|c|}{$2(4.8 \%)$} & $7(16.7 \%)$ & \multicolumn{2}{|c|}{$4(9.5 \%)$} & $16(38.1 \%)$ \\
\hline \multirow[t]{2}{*}{ Alto } & \multicolumn{2}{|c|}{$3(7.1 \%)$} & $9(21.4 \%)$ & \multicolumn{2}{|c|}{$3(7.1 \%)$} & $8(19.0 \%)$ & \multicolumn{2}{|c|}{$5(11.9 \%)$} & $13(31.0 \%)$ \\
\hline & \multicolumn{3}{|c|}{$\left(\chi^{2}=0.108 ; p<0.947\right)$} & \multicolumn{3}{|c|}{$\left(\chi^{2}=0.098 ; p<0.952\right)$} & \multicolumn{3}{|c|}{$\left(\chi^{2}=0.654 ; p<0.718\right)$} \\
\hline Libra guardias & \multicolumn{2}{|c|}{ No } & Sí & \multicolumn{2}{|c|}{ No } & Sí & \multicolumn{2}{|c|}{ No } & Sí \\
\hline Bajo & \multicolumn{2}{|c|}{$2(4.8 \%)$} & $18(42.9 \%)$ & \multicolumn{2}{|c|}{$2(4.8 \%)$} & $20(\%)$ & \multicolumn{2}{|c|}{$0(0 \%)$} & $4(9.5 \%)$ \\
\hline Medio & \multicolumn{2}{|c|}{$0(0 \%)$} & $10(0 \%)$ & \multicolumn{2}{|c|}{$1(2.4 \%)$} & $8(19 \%)$ & \multicolumn{2}{|c|}{$2(4.8 \%)$} & $18(42.8 \%)$ \\
\hline \multirow[t]{2}{*}{ Alto } & \multicolumn{2}{|c|}{$1(2.4 \%)$} & $11(26.2 \%)$ & \multicolumn{2}{|c|}{$0(0 \%)$} & $11(26.2 \%)$ & $1(2$ & $4 \%)$ & $17(40.5 \%)$ \\
\hline & & $\chi^{2}=1.727$ & $<0.222)$ & & $\chi^{2}=1.932 ; p$ & 0.381) & & $t^{2}=1.029 ; p$ & $<0.598)$ \\
\hline $\begin{array}{l}\text { Año der } \\
\text { esidencia }\end{array}$ & & 81 & $\mathrm{R} 2$ & & R1 & $\mathrm{R} 2$ & & 1 & $\mathrm{R} 2$ \\
\hline Bajo & & $26.8 \%)$ & $9(21.4 \%)$ & 14( & $33.3 \%)$ & $8(19 \%)$ & $3(7$ & $1 \%)$ & $1(2.4 \%)$ \\
\hline Medio & & $4.3 \%)$ & $4(9.5 \%)$ & 5( & $1.9 \%)$ & $4(9.5 \%)$ & 15 & $5.7 \%)$ & $5(11.9 \%)$ \\
\hline Alto & & 19\%) & 4 (9.5\%) & & $4.3 \%)$ & $4(11.9 \%)$ & $7(1$ & $.7 \%)$ & $11(26.2 \%)$ \\
\hline & & $\chi^{2}=0.426$ & $<0.407)$ & & $\chi^{2}=0.326 ; p$ & $<0.849)$ & & $t^{2}=6.216 ; p$ & $<0.045)$ \\
\hline Especialidad & Médica & Quirúrgica & $\begin{array}{c}\text { Médica } \\
\text {-quirúrgica }\end{array}$ & Médica & Quirúrgica & $\begin{array}{l}\text { Médica } \\
\text {-quirúrgica }\end{array}$ & Médica & Quirúrgica & $\begin{array}{l}\text { Médica } \\
\text {-quirúrgica }\end{array}$ \\
\hline Bajo & $16(38.1 \%)$ & $2(4.8 \%)$ & $2(4.8 \%)$ & $17(40.5 \%)$ & $3(7.4 \%)$ & $2(4.8 \%)$ & $4(9.5 \%)$ & $0(0 \%)$ & $0(0 \%)$ \\
\hline Medio & $9(21.4 \%)$ & $0(0 \%)$ & $1(2.4 \%)$ & $7(16.7 \%)$ & $1(2.4 \%)$ & $1(2.4 \%)$ & $12(28.6 \%)$ & $4(9.5 \%)$ & $4(9.5 \%)$ \\
\hline Alto & $7(16.7 \%)$ & $3(7.1 \%)$ & $2(4.8 \%)$ & $8(19 \%)$ & $1(2.4 \%)$ & 2 (4.8\%) & $16(38.1 \%)$ & $1(2.4 \%)$ & $1(2.4 \%)$ \\
\hline & $\left(\chi^{2}\right.$ & $4.875 ; p<0$ & 300) & $\left(\chi^{2}=\right.$ & $0.639 ; p<0$ & 959) & $\left(\chi^{2}=\right.$ & 7.698; $p<0$. & 103) \\
\hline
\end{tabular}

R1: residente de primer año; R2: residente de segundo año.

tarea y un AS reducido ${ }^{9}$. Estos resultados parecen apuntar a un problema estructural de los servicios de urgencias ${ }^{17}$ que se puede agravar por la falta de medios técnicos y humanos ${ }^{18}$.

Es posible que las altas EP encontradas se deban al déficit de habilidades clínicas o a la mala autocalibración de las habilidades y los conocimientos reales de los MIR. En cualquier caso, se podría pensar que los residentes están expuestos a una serie de demandas y contingencias laborales para las que pueden no estar adecuadamente formados, lo que explicaría por qué el $90 \%$ de ellos presenta unas puntuaciones desfavorables (Fig. 1). La formación que se recibe durante el grado, y posteriormente para el examen nacional de acceso a MIR, es eminentemente teórica ${ }^{19,20}$. Cuando los residentes se incorporan al hospital, ellos mismos manifiestan déficits importantes en habilidades clínicas, de comunicación, exploratorias, de interpretación de pruebas y de organización (redacción de informes, recetas, entre otros) $)^{21}$.

Así pues, la discontinuidad existente entre la formación durante el grado, la preparación para el examen MIR y el comienzo de la especialidad puede situar a los residentes en una situación psicosocial de alta vulnerabilidad al estrés y a las EP.

El AS de iguales y superiores es fundamental para la adecuada integración y estabilización de los residentes en los servicios de urgencias debido a su papel modulador con el estrés y las EP. Por eso, los resultados obtenidos en la tabla 1 son preocupantes, ya que muestran las preferencias de los residentes 
Gaceta Médica de México. 2017;153

Tabla 3. Correlaciones parciales de Pearson entre la EEP, el MBI y el ISTAS21 corregidas por edad $(n=42)$

\begin{tabular}{|c|c|c|c|c|c|c|c|c|c|}
\hline & $\begin{array}{l}\text { Agotamiento } \\
\text { emocional }\end{array}$ & Despersonalización & $\begin{array}{c}\text { Realización } \\
\text { personal }\end{array}$ & $\begin{array}{l}\text { Exigencias } \\
\text { psicológicas }\end{array}$ & $\begin{array}{l}\text { Control del } \\
\text { puesto }\end{array}$ & $\begin{array}{l}\text { Apoyo } \\
\text { social }\end{array}$ & $\begin{array}{l}\text { Estima } \\
\text { personal }\end{array}$ & $\begin{array}{c}\text { Doble } \\
\text { presencia }\end{array}$ & $\begin{array}{l}\text { Inseguridad } \\
\text { futuro }\end{array}$ \\
\hline EEP & $0.573^{*}$ & $0.520^{*}$ & $-0.306^{\dagger}$ & $0.316^{\dagger}$ & $-0.301^{\dagger}$ & $-0.370^{+}$ & -0.172 & 0.156 & 0.103 \\
\hline Agotamiento emocional & & $0.754^{*}$ & $-0.398^{*}$ & $0.535^{\star}$ & $-0.360^{+}$ & $-0.445^{*}$ & -0.443 & 0.103 & 0.224 \\
\hline Despersonalización & & & -0.240 & 0.270 & -0.175 & -0.246 & -0.186 & 0.116 & -0.038 \\
\hline Realización personal & & & & -0.028 & 0.271 & 0.226 & 0.300 & -0.264 & -0.193 \\
\hline $\begin{array}{l}\text { Exigencias } \\
\text { psicológicas }\end{array}$ & & & & & $-0.390^{\dagger}$ & $-0.534^{*}$ & $-0.531^{*}$ & -0.198 & $0.327^{\dagger}$ \\
\hline Control del puesto & & & & & & $0.623^{*}$ & $0.619^{*}$ & -0.049 & -0.061 \\
\hline Apoyo social & & & & & & & $0.692^{*}$ & -0.070 & -0.060 \\
\hline Estima personal & & & & & & & & -0.165 & -0.126 \\
\hline Doble presencia & & & & & & & & & 0.075 \\
\hline
\end{tabular}

Tabla 4. Regresión múltiple "por pasos sucesivos» utilizando las dimensiones del CopSoQ-ISTAS21 como predictores y las dimensiones del MBI como criterio $(n=42)$

\begin{tabular}{|c|c|c|c|c|c|c|}
\hline \multirow[b]{3}{*}{ Predictor } & \multirow{2}{*}{\multicolumn{2}{|c|}{$\begin{array}{c}\begin{array}{c}\text { Agotamiento emocional } \\
(n=42)\end{array} \\
R^{2} \text { corregida: } 0.443\end{array}$}} & \multirow{2}{*}{\multicolumn{2}{|c|}{$\begin{array}{c}\begin{array}{c}\text { Despersonalización } \\
(n=42)\end{array} \\
R^{2} \text { corregida: } 0.199\end{array}$}} & \multirow{2}{*}{\multicolumn{2}{|c|}{$\begin{array}{c}\begin{array}{c}\text { Realización personal } \\
(n=42)\end{array} \\
R^{2} \text { corregida: } 0.262\end{array}$}} \\
\hline & & & & & & \\
\hline & $\begin{array}{c}\text { Beta } \\
\text { estandarizado (IC) }\end{array}$ & $p$ & $\begin{array}{c}\text { Beta } \\
\text { estandarizado } \\
\text { (IC) }\end{array}$ & $p$ & $\begin{array}{c}\text { Beta } \\
\text { estandarizado } \\
\text { (IC) }\end{array}$ & $p$ \\
\hline $\begin{array}{l}\text { Exigencias } \\
\text { psicológicas }\end{array}$ & $\begin{array}{c}0.397 \\
(0.412-2.140)\end{array}$ & 0.003 & 0.115 & 0.440 & $\begin{array}{c}0.390 \\
(0.016-1.376)\end{array}$ & 0.045 \\
\hline Control del trabajo & -0.084 & 0.533 & -0.020 & 0.892 & 0.090 & 0.637 \\
\hline Apoyo social & 0.052 & 0.686 & -0.092 & 0.514 & 0.016 & 0.944 \\
\hline Inseguridad & -0.090 & 0.544 & -0.058 & 0.702 & -0.232 & 0.135 \\
\hline Doble presencia & 0.106 & 0.396 & 0.032 & 0.823 & 0.069 & 0.674 \\
\hline Estima personal & -0.204 & 0.156 & -0.091 & 0.520 & $\begin{array}{c}0.431 \\
(0.198-1.959)\end{array}$ & 0.018 \\
\hline EEP & $\begin{array}{c}0.451 \\
(0.358-1.257)\end{array}$ & 0.001 & $\begin{array}{c}0.520 \\
(0.232-0.775)\end{array}$ & 0.001 & $\begin{array}{c}-0.325 \\
(-0.610-1.376)\end{array}$ & 0.038 \\
\hline
\end{tabular}

IC: intervalo de confianza.

por consultar antes a sus compañeros que a los facultativos, y cuando consultan, se iguala la percepción de resolución ofrecida por un igual o un superior. Llama la atención que los residentes se sienten significativamente más respaldados por sus compañeros que por los facultativos, lo que podría explicar parte del AS desfavorable que muchos manifiestan percibir (Fig. 1). Estos datos no se encuentran en consonancia cuando se habla de facultativos de atención primaria y son encuestados sobre su satisfacción laboral y los aspectos de mejora en el puesto de trabajo ${ }^{22}$.

Además, los resultados reflejan que un porcentaje inferior al $40 \%$ de los residentes tiene niveles medios 0 altos de burnout. Sin embargo, la ausencia de un criterio consensuado sobre el burnout y las diversas posibilidades metodológicas dificultan las comparaciones entre estudios ${ }^{7,23}$. Los valores medios obtenidos en nuestra muestra en las distintas dimensiones del MBI son convergentes con otros estudios ${ }^{24}$. 
Las variables sociodemográficas no arrojan diferencias, ya que el sexo, el año de residencia, el tipo de especialidad, haber sufrido agresiones 0 amenazas y descansar o no el día posterior a la guardia no presentan diferencias para las dimensiones del MBI ni para las del ISTAS21.

En este trabajo no se ha encontrado relación entre el número de horas de guardia y el burnout, aunque otros autores sí la han establecido $0^{6}$. Hay que señalar que nuestros residentes han indicado un número medio de 68.31 horas al mes, lo que supone aproximadamente tres guardias mensuales. No obstante, a algunos residentes habría que sumarles las guardias propias de su especialidad. En cualquier caso, parece apreciarse que los niveles de burnout de los profesionales sanitarios siguen siendo especialmente elevados, por lo que es necesario apuntar a otras variables psicosociales que nos ayuden a predecirlo. Esta apreciación parece estar relacionada con algunos trabajos que no han descrito reducciones en el grado de burnout pese a la reducción de las horas de trabajo ${ }^{25}$.

Como se ha puesto de manifiesto en otros trabajos, encontramos una relación entre el estrés y el burnout ${ }^{25,26}$, especialmente en lo relativo al AE. Además, la tabla 3 muestra las importantes correlaciones entre el estrés y las EP, el CT y el AS. Esto evidencia el papel modulador del estrés con relación a los riesgos asociados al puesto de trabajo, y la necesidad de realizar intervenciones que mejoren las condiciones laborales.

Por último, parece relevante mencionar que el 38\% de los residentes ha experimentado algún tipo de agresión o amenaza por parte de los pacientes. Estos datos vienen a refrendar la preocupante situación en que se encuentra el colectivo sanitario en general y, en particular, el colectivo médico ${ }^{27,28}$. Algunos autores han asociado precisamente los altos niveles de ansiedad, el cansancio emocional y la despersonalización con las agresiones físicas ${ }^{29}$.

Las limitaciones propias del trabajo han sido su tamaño muestral y la tasa final de participación, que son problemas comunes a la mayoría de los estudios sobre este tema. Se ha realizado un estudio unicéntrico, aunque las características de nuestro hospital universitario son similares a las del ámbito nacional, por lo que cabría pensar en una posible generalización de los resultados. En futuros trabajos sería interesante abordar esta realidad desde un estudio multicéntrico y seguir explorando los constructos utilizados y otras dimensiones psicológicas que puedan predecir el burnout. Se evidencia la necesidad de aumentar el apoyo a los residentes por parte de los facultativos. Igualmente, se sugiere la puesta en marcha de programas formativos en habilidades con las que mejorar la transición del grado a la residencia, tal como se hace en otros países de nuestro entorno ${ }^{30}$.

\section{Agradecimientos}

A los médicos internos residentes del Hospital Clínico San Cecilio (Granada, España) que han comprendido la importancia y la necesidad del estudio.

\section{Conflicto de intereses}

No existen relaciones financieras, laborales ni de otra índole que puedan constituirse como conflicto de intereses respecto del presente trabajo.

\section{Bibliografía}

1. Ministerio de Sanidad, Servicios Sociales e Igualdad. Un total de 35.320 titulados optan mañana a una de las 7.527 plazas de formación sanitaria especializada de la convocatoria 2014-2015. Madrid; 2015. (Consultado el 15 de febrero de 2015.) Disponible en: http://www.msssi.gob.es/ gabinete/notasPrensa.do?id=3541.

2. Gaceta Médica. En España hay $30.051 \mathrm{MIR}$, un $3,76 \%$ menos que el año pasado. Madrid; 2015. (Consultado el 1 de marzo de 2015.) Disponible en: http//www.gacetamedica.com; http://goo.gl/qC5XRU.

3. Gutiérrez Díez MC, Santamaría Pablos A, Delgado Diego A, Moreno Marín JA, Monasterio Rentaría AM, Landaluce Fuentes M, et al. Desgaste profesional: calidad de las relaciones interpersonales en los equipos de trabajo. Emergencias. 2008;20:73-4.

4. Moreno-Millán E. Estrés ocupacional en los profesionales de la medicina aguda. Emergencias. 2007;19:151-3.

5. Fernández-Prada M, González-Cabrera J, Torres F, Iribar-lbabe C, Peinado JM. Calidad de vida relacionada con la salud en una muestra de médicos internos residentes que realizan guardias en un servicio de urgencias: una perspectiva de género. Rev Med Chile. 2014;142:193-8.

6. Fonseca M, Sanclemente G, Hernández C, Visiedo C, Bragulat E, Miró O. Residentes, guardias y síndrome de burnout. Rev Clin Esp. 2010;210:209-15.

7. Grau A, Flichtentrei D, Suñer R, Prats M, Braga M. Influencia de factores personales, profesionales y transnacionales en el síndrome de burnout en personal sanitario hispanoamericano y español (2007). Rev Esp Salud Publica. 2009;83:215-30.

8. Agencia Europea para la Seguridad y la Salud en el Trabajo. La semana europea para la seguridad y la salud en el trabajo. Bruselas; 2015. (Consultado el 1 de marzo de 2015.) Disponible en: https://www.healthy-workplaces.eu/es/; https://www.healthy-workplaces.eu/es/campaign-partners/national-focal-points.

9. González-Cabrera J, Fernández-Prada M, Molina-Ruano R, Blazquez A Guillén-Solvas J, Peinado JM. Psychosocial risk at work, self-perceived stress, and salivary cortisol level in a sample of emergency physicians in Granada. Emergencias. 2012;24:101-6.

10. Ghaddar A, Ronda E, Nolasco A, Álvares N, Mateo I. Exposure to psychosocial risks at work in prisons: does contact with inmates matter? A pilot study among prison workers in Spain. Stress Health. 2011; 27:170-6.

11. Bermúdez RM, Martínez-Arquero G, Barón FJ, Hernández-Mendo A. An interdisciplinary approach to teachers' voice disorders and psychosocial working conditions. Folia Phoniatr Logop. 2010;62:24-34.

12. Cohen S, Herbert TB. Health psychology: psychological factors and physical disease from the perspective of human psychoneuroimmunology. Annu Rev Psychol. 1996;47:113-42. 
13. Trujillo HM, González-Cabrera J. Propiedades psicométricas de la versión española de la "Escala de estrés percibido" (EEP). Psicol Conductual. 2007;15:457-77.

14. Moncada S, Llorens C, Font A, Galtés F, Giné A. Exposición a riesgos psicosociales entre la población asalariada en España (2004-05): valores de referencia de las 21 dimensiones del cuestionario ISTAS21 COP. SOQ. Rev Esp Salud Publica. 2008;82:667-75.

15. Gil-Monte PR, Peiró JM. Validez factorial del Maslach Burnout Inventory en una muestra multiocupacional. Psicothema. 1999;11:679-89.

16. Karasek R, Theorell, T. Healthy work. Stress, productivity, and the reconstruction of working life. New York: Basic Books; 1990.

17. Moreira JM, Álvarez, MC. Clima organizacional y estrés en una unidad de alto riesgo. Emergencias. 2002;14:6-12.

18. Sánchez M, Salgado E, Miró O. Mecanismos organizativos de adaptación y supervivencia de los servicios de urgencias. Emergencias. 2008;20:48-53.

19. Peinado-Herreros JM. Definición de las competencias que debe adquirir el futuro médico. Formación de los profesores. Educ Med. 2008; 11:47-51

20. González de Dios P, Pollanco I, Díaz CA. De las facultades de medicina a la residencia de pediatría, pasando por el examen de médico interno residente: ¿algo debe cambiar? Resultados de una encuesta en residentes de pediatría de cuarto año. An Pediatr (Barc). 2009;70:467-76.

21. Llupià A, Costas L, Grau J, Trilla A. Conocimientos, capacidades e intereses de los médicos internos y residentes (MIR) al incorporarse al hospital. Med Clin (Barc). 2009;133:107-11.
22. Pérez-Ciordia I, Guillén-Grima F, Brugos A, Aguinaga Ontoso I. Satisfacción laboral y factores de mejora en profesionales de atención primaria. An Sist Sanit Navar. 2013;36:2453-62.

23. Grau-Martín A, Suñer-Soler R. Síndrome de burnout y residentes: reflexiones sobre la magnitud del fenómeno. Rev Clin Esp. 2011; 211:112-3.

24. Suñer-Soler R, Grau-Martín A, Font-Mayolas S, Gras ME, Bertran C, Sullman MJ. Burnout and quality of life among Spanish healthcare personnel. J Psychiatr Ment Health Nurs. 2013;20:305-13.

25. Gelfand DV, Podnos YD, Carmichael JC, Saltzman DJ, Wilson SE, Williams RA. Effect of the 80-hour workweek on resident burnout. Arch Surg. 2004;139:933-8.

26. Graham J, Potts HW, Ramirez AJ. Stress and burnout in doctors. Lancet. 2002;360:1975-6.

27. Miret C, Larrea A. El profesional en urgencias y emergencias: agresividad y burnout. An Sist Sanit Navar. 2010;33(Supl 1):193-201.

28. Martínez-Jarreta B, Gascón S, Santed MA, Goicochea J. Análisis médico-legal de las agresiones a profesionales sanitarios. Aproximación a una realidad silenciosa y a sus consecuencias para la salud. Med Clin (Barc). 2007;128:307-10.

29. Gascón S, Martínez-Jarreta B, López Verdejo MA, Diana I, López-Torres J, Castellano M. Respuestas desadaptativas al estrés derivadas de agresiones a profesionales sanitarios. Rev SEMST. 2008;3:103-5.

30. Van Hell EA, Kuks JBM, Borleffs JCC, Cohen-Schotanus J. Alternating skills training and clerkships to ease the transition from preclinical to clinical training. Med Teach. 2011;33:689-96. 